\title{
Función sensorial y dependencia en adultos mayores con enfermedad crónica
}

\author{
Tirso Duran-Badillo ${ }^{1}$ (D); Martha Elba Salazar- Barajas ${ }^{2 *}$ (D); Perla Lizeth Hernández Cortés ${ }^{3}$ (D); \\ Milton Carlos Guevara-Valtier 4 (D); Gustavo Gutiérrez-Sánchez 5 (D)
}

\begin{abstract}
RESUMEN
Introducción: Con el envejecimiento se presentan cambios en los sentidos y en consecuencia, alteraciones en la función del gusto y olfato que influyen en la descompensación de enfermedades crónicas. Objetivo: conocer la función sensorial y su asociación con la dependencia para realizar Actividades básicas de la vida diaria e Instrumentales en adultos mayores con enfermedad crónica. Metodología: Estudio correlacional en una muestra de 96 participantes. Instrumentos utilizados: Carta Snellen, Audiómetro Manual, monofilamento de Semmes-Weinstein, prueba de aromas de Gustos Básicos e Índice de Barthel. El análisis estadístico implicó estadística descriptiva e inferencial, correlación de Spearman, U de Mann-Whitney. Resultados: El 71\% de los participantes presentó alteración visual. $48 \%$ obtuvo audición normal en oído izquierdo y $43 \%$ oído derecho, $24 \%$ calificó con alteración táctil del pie izquierdo y $30 \%$ en pie derecho. Alteración de olfato y gusto en $77 \%$. Presentaron dependencia en actividades Básicas (ABVD) 40\%, $24 \%$ en Instrumentadas (AIVD). Se identificó relación entre el tacto en ambos pies y agudeza auditiva con ABVD $(p=<.05)$. La edad y AIVD $(p=<.001)$ agudeza tacto pie izquierdo $(p=<.031)$, auditiva $(p=<.005)$, olfato $(p=.018)$ y gusto $(p=<.047)$. La dependencia en ABVD no presentó diferencia en la prueba con anteojos $(U=146.000 ; p=<.754)$ y $\sin$ anteojos $(U=127.500$; $p=.079)$; de igual manera en AIVD con anteojos $(U=115.000 ; p=<.079)$ y sin anteojos $(U=160.000$; $p=.202)$. Conclusión: A mayor función en los sentidos de tacto y oído, menor dependencia para realizar las ABVD y a mayor función del tacto, oído, olfato y gusto menor dependencia en las AIVD.
\end{abstract}

Palabras clave: Sensación; Marcha; Cognición; Actividades cotidianas; Adulto mayor (DeCs).

\footnotetext{
${ }^{1}$ Enfermero, Master, Docente. Unidad Académica Multidisciplinaria, Matamoros. Universidad Autónoma de Tamaulipas (México).

${ }^{2}$ Enfermera, Doctora, Docente. Unidad Académica Multidisciplinaria, Matamoros. Universidad Autónoma de Tamaulipas (México).

${ }^{3}$ Enfermera, Doctora, Docente. Facultad de Enfermería. Universidad Autónoma de Nuevo León. (México).

${ }^{4}$ Enfermero, Doctor, Docente. Facultad de Enfermería. Universidad Autónoma de Nuevo León. (México).

${ }^{5}$ Psicólogo, Doctor, Docente. Unidad Académica Multidisciplinaria, Matamoros. Universidad Autónoma de Tamaulipas (México).
}

*Autor para correspondencia: marthasalbar@yahoo.com.mx

Recibido: $21 / 01 / 2020$

Aceptado: $24 / 08 / 2020$

Cómo citar este artículo

Duran-Badillo T, Salazar- Barajas ME, Hernández Cortés PL, Guevara-Valtier MC, Gutiérrez-Sánchez G . Función sensorial y dependencia en adultos mayores con enfermedad crónica. SANUS. 2020; (15): 1-11. [Acceso dd $\underline{\mathrm{mm}} \underline{\text { aal; }}$ Disponible en: $\underline{\text { URL/DOI. }}$ 


\title{
Sensory function and dependence in older adults with chronic disease
}

\begin{abstract}
Introduction: With aging there are changes in the senses and consequently, alterations in the function of taste and smell that influence the decompensation of chronic diseases. Objective: Know the sensory function and its association with dependence to perform basic and instrumental daily life activities in older adults with chronic diseases. Methodology: Correlational study in a sample comprised by 96 participants. The Instruments used were: Snellen Letter, Manual Hearing meter, Semmes-Weinstein monofilament, flavor test, Basic Tastes, and Barthel Index. The statistic analysis implied the use of descriptive and inferential statistics, Spearman Correlation, and Mann-Whitney U Test. Results: Seventy one percent $(71 \%)$ had visual impairment; $48 \%$ got normal hearing in the left ear and $43 \%$ in the right ear; $24 \%$ rated with tactile alteration in the left foot and $30 \%$ with tactile alteration in the right foot; $77 \%$ presented alteration of smell and taste. Forty percent (40\%) had dependence on Basic Activities (ABVD by its acronym in Spanish) and $24 \%$ in Instrumental Activities (AIVD by its acronym in Spanish). The relationship between touch in both feet and auditory acuity was identified with ABVD $(p=<.05)$. Age and AIVD $(p=<.001)$, sharp touch in the left foot $(p=<.031)$, auditory $(p=<.005)$, smell $(p=<.018)$, and taste $(p=<.047)$. The dependence on ABVD had no difference in the test with eyeglasses $(U=146,000 ; p-$ $754)$ and without eyeglasses $(U=127,500 ; p-079) ;$ AIVD with eyeglasses $(U=115,000 ; p-079)$ and without eyeglasses $(U=160,000 ; p-202)$. Conclusions: The greater the function in the touch and hearing senses, the less the dependence to perform ABVDs and the greater the functions in the touch, hearing, and smell senses, the less the dependence on AIVDs.
\end{abstract}

Keywords: Sensation; Gait; Cognition; Daily Life Activities; Older Adult (DeCS). 


\title{
Função sensorial e dependência em idosos com doença crônica
}

\begin{abstract}
ABSTRATO
Introdução: Com o envelhecimento há mudanças nos sentidos e, conseqüentemente, alterações na função do paladar e do olfato que influenciam a descompensação das doenças crônicas. Objetivo: Conhecer a função sensorial e sua associação com a dependência para realizar Atividades Básicas da Vida Cotidiana e Instrumental em idosos com doença crônica. Metodologia: Estudo correlacionado em uma amostra de 96 participantes. Instrumentos utilizados: Gráfico Snellen, Audiometer Manual, Monofilamento Semmes-Weinstein, Teste de Smell de Sabores Básicos e Índice Barthel. A análise estatística envolveu estatísticas descritivas e inferenciais, correlação de Spearman, Mann-Whitney U. Resultados: $71 \%$ dos participantes apresentaram deficiência visual. $48 \%$ obtiveram audição normal no ouvido esquerdo e $43 \%$ ouvido direita, $24 \%$ qualificadas com alteração tátil do pé esquerdo e $30 \%$ no pé direito. Olfato e paladar perturbam em $77 \%$. Eles tinham dependência de Atividades Básicas (ABVD) $40 \%, 24 \%$ em Instrumentos (AIVD). A relação foi identificada entre o toque em ambos os pés e a acuidade auditiva com a ABVD $(p=<05)$. Idade e AIVD $(p=<001)$ toque agudo do pé esquerdo ( $p=$ $<031)$, auditivo $(p=<.005)$, cheiro $(p=<.018)$ e paladar $(p=<.047)$. A dependência da ABVD não mostrou diferença no teste com óculos $(U=146.000 ; p=<.754)$ e sem óculos $(U=127.500 ; p=<.079)$; da mesma forma em AIVD com óculos ( $U=115.000 ; p=<.079)$ e sem óculos $(U=160.000 ; p=<.202)$. Conclusão: A maior função nos sentidos de tato e audição, menor dependência para realizar ABVDs e maior função de toque, audição, olfato e menos dependência dos AIVDs. .
\end{abstract}

Palavras-chave: Sensação; Marcha; Cognição; Atividades da vida; Idoso (DeCS). 


\section{INTRODUCCIÓN}

Las enfermedades crónicas son un problema de salud a nivel mundial. (1) En México las prevalencias más altas de diabetes mellitus, hipertensión arterial y dislipidemias corresponden a la población de adultos, (2) conforme pasa la edad se presentan otros problemas como la alteración sensorial y por consecuencia la disminución en la habilidad para ejecutar actividades básicas e instrumentales de la vida cotidiana. ${ }^{(3)}$

La función sensorial es la capacidad de recibir e interpretar sensaciones del exterior a través de los órganos de los sentidos y la literatura indica que con el envejecimiento se presentan importantes cambios en cada uno de ellos. La alteración de la agudeza olfativa y gustativa, es importante estudiarlas debido a que alteran la capacidad de degustar y preparar los alimentos, además se relacionan con la inseguridad (consumo de alimentos descompuestos, dejar abierta la perilla del gas, etc.) y la higiene personal. ${ }^{(4)}$ Se ha documentado que las personas con daño en la agudeza olfativa tienen problemas para percibir los sabores ${ }^{(5)}$ en consecuencia, las alteraciones en la función del gusto y olfato influyen en la descompensación de enfermedades crónicas como diabetes mellitus, hipertensión, entre otras; ya que las personas al no percibir los sabores pueden incrementar el consumo de azúcar o sal en los alimentos. ${ }^{(6)}$

El sentido del tacto, disminuye por el número de receptores y cambia su integridad estructural, por lo que al no percibir las cualidades de los objetos como la presión, temperatura, dureza, asperidad o suavidad, se incrementa el riesgo de sufrir lesiones. En los adultos mayores con enfermedades crónicas como la diabetes mellitus, las lesiones conllevan a complicaciones que pueden culminar con la pérdida de algún miembro de extremidades superiores 0 inferiores. ${ }^{(7)}$

Por otro lado, en el oído los sonidos de alta frecuencia de $2000 \mathrm{~Hz}$ y superiores son los primeros que se pierden, resultando en alteración de la comunicación y aislamiento de los adultos mayores. La comunicación es importante para realizar todo tipo de actividades básicas y otras como hacer compras, entre estas la selección de alimentos para el control de su enfermedad crónica, hablar por teléfono y trasladarse de un lugar a otro. Sin duda el sentido de la vista, es el que mayor impacta en las personas mayores. (3) Se ha documentado que cuando se deteriora el sentido de la vista, la dependencia del adulto mayor se incrementa debido a que afecta todas las actividades. ${ }^{(8)}$ La Encuesta Nacional de Salud y Nutrición (2018) (2) reportó que en pacientes con diabetes mellitus el principal problema que se presentó fue la visión disminuida, seguida de la pérdida de sensibilidad en la planta de los pies.

La revisión de la literatura, ha mostrado poca evidencia científica en la que se estudia la función sensorial y la dependencia funcional, por lo que, de acuerdo con lo ya descrito se consideró importante realizar un estudio que permita conocer la agudeza visual, olfativa, gustativa, táctil, auditiva y su asociación con la dependencia para realizar las actividades básicas e instrumentales de la vida diaria, además explorar si la dependencia para realizar las actividades básicas de la vida diaria (ABVD) y actividades instrumentadas de la vida diaria (AIVD) es diferente en adultos mayores con alteración visual en comparación con aquellos que tienen agudeza visual normal. El objetivo de este trabajo fue conocer la función sensorial y su asociación con la dependencia para realizar las ABVD e Instrumentales en adultos mayores con enfermedad crónica.

Se espera que los resultados de este trabajo contribuyan al incremento del conocimiento de estos fenómenos y sirvan de fundamento para proponer intervenciones dirigidas a retrasar la alteración de la función sensorial en los adultos mayores con enfermedades crónicas.

\section{METODOLOGÍA}

Estudio descriptivo y correlacional en una población urbana que acude a la consulta en un Centro de Salud en Monterrey, Nuevo León, México. La muestra estuvo conformada por 96 
adultos mayores que reportaron padecer alguna enfermedad crónica, identificados a través de un muestreo no probabilístico por conveniencia. Fueron considerados como criterios de inclusión: adultos mayores con capacidad de escuchar y contestar coherentemente al entrevistador, se excluyeron aquellos que reportaron enfermedad menor a un año.

Los adultos mayores que conformaron la muestra de estudio indicaron que: $37.5 \%(f=36)$ padece diabetes mellitus, el $34.4 \% \quad(f=33)$ hipertensión arterial, el 15.6\% ( $f=15)$ diabetes mellitus, hipertensión arterial e hipercolesterolemia o hipertrigliceridemia y el $12.5 \% \quad(f=12) \quad$ hipercolesterolemia o hipertrigliceridemia.

La función sensorial incluyó la valoración de la agudeza visual, auditiva, táctil, olfativa y gustativa. La agudeza visual se midió con la Carta Snellen de letras y/o dibujos. Se consideró visión normal cuando los parámetros resultaron en 20/15 o 20/20. La agudeza auditiva se midió con un Audiómetro Manual $232^{\mathrm{TM}}$ de la marca WelchAllyn. Los resultados globales se agruparon en seis niveles de audición para ambos oídos: 1) de -10 a 26 dB audición normal, 2) de 27 a $40 \mathrm{~dB}$ pérdida auditiva leve, 3) de 41 a $55 \mathrm{~dB}$ pérdida auditiva moderada, 4) de 56 a $70 \mathrm{~dB}$ pérdida auditiva moderadamente severa, 5) de 71 a 90 severa y 6) $>91 \mathrm{~dB}$ pérdida auditiva profunda. Para fines de las correlaciones se reportó el valor en $\mathrm{dB}$ en el que el sujeto indicó escuchar. Un mayor puntaje representa mayor deterioro auditivo. ${ }^{(9)}$

Para la agudeza del tacto en los adultos mayores se valoró la sensibilidad del dorso y planta de los pies con un monofilamento de Semmes-Weinstein de $10 \mathrm{gr}$. Se tocó en un punto del dorso y nueve puntos de la planta en cada pie. Se consideró el total de puntos percibidos por el sujeto ( 0 a 10$)$, a mayor cantidad de puntos percibidos mayor sensibilidad, siendo normal cuando reportó los 10 puntos.

La agudeza olfativa fue medida con la prueba de aromas, se utilizaron cinco aromas naturales (comino, pimienta, anís, canela y romero); las sustancias se cubrieron con algodón y los recipientes se etiquetaron con el nombre que correspondía. En otros recipientes se colocaron las mismas sustancias y los recipientes se etiquetaron con códigos. Al sujeto se le dio la indicación de oler de izquierda a derecha cada sustancia etiquetada con nombres y que memorizaran el aroma, posteriormente se le dio a oler café para neutralizar los aromas y se le pidió que oliera los recipientes que se encontraban en los recipientes codificados y dijera a qué sustancia correspondía. Se hizo una sumatoria de los aromas distinguidos, a mayor puntuación se consideró mejor agudeza olfativa.

La agudeza gustativa fue medida con la Prueba de Gustos Básicos con método de selección de Caul, que mide la habilidad para reconocer cuatro gustos básicos (dulce, salado, ácido y amargo). Se utilizó sacarosa (16 $g / l)$, cloruro de sodio $(5 \mathrm{~g} / \mathrm{l})$, ácido cítrico $(1 \mathrm{~g} / \mathrm{l})$ y agua quina (.5, sin diluir). Las sustancias fueron pesadas en una báscula analítica de la marca AND, serie HR200, posteriormente, fueron diluidas en envases con un litro de agua. Las sustancias diluidas se colocaron en recipientes con $20 \mathrm{ml}$, los recipientes se codificaron del 1 al 4 de acuerdo al tipo de sustancia y se presentaron a cada sujeto para que identificara el sabor de cada una. Se dio un bote con agua para que el sujeto se enjuagara o tomara agua las veces que fuera necesario antes o después de probar cada sustancia. Una vez realizada la prueba se hizo una sumatoria de los sabores que distinguió, a mayor puntuación se consideró mejor agudeza gustativa.

Para medir la dependencia se utilizó el Índice de Barthel para las actividades básicas de la vida diaria (ABVD) con alpha de Cronbach de .86-.92, se consideró independiente cuando el adulto mayor resultó con 100 puntos (10). Las actividades instrumentales de la vida diaria (AIVD) fueron evaluadas con el índice de Lawton y Brody, con una fiabilidad intra clase de 0.95, (11) se consideró independiente cuando el sujeto reportó realizar de manera autónoma las ocho actividades que evalúa el instrumento.

Los datos fueron procesados y analizados en el programa estadístico Statistical Package for the Social Sciences (SPSS) versión 20 para Windows. Se utilizó estadística descriptiva e inferencial. Se empleó la prueba de normalidad de 
Kolmogorov Smirnov con corrección de Lilliefors y en función de los resultados se utilizó el coeficiente de correlación de Spearman y $U$ de Mann-Whitney. Se contó con la aprobación de los comités de Investigación y Ética en Investigación de la Facultad de Enfermería de la Universidad Autónoma de Nuevo León, del comité de la Dirección de Enseñanza, Investigación en Salud y Calidad de la Secretaría de Salud de Nuevo León y con la autorización de la Secretaría de Salud para hacer la invitación a participar a los adultos mayores. El estudio se clasificó dentro de la categoría de riesgo mínimo ya que se realizaron pruebas de funcionalidad sensorial.

\section{RESULTADOS}

Participaron 96 sujetos, el $79.2 \%$ ( $f=76)$ mujeres, en promedio tenían 69.33 años de edad ( $D E=$ 6.53), y 9.02 años de escolaridad ( $D E=4.69)$, reportaron consumir en promedio 3.31 medicamentos $(D E=2.01)$. Ninguno de los participantes presentó tapón auditivo o exceso de cerumen. En la tabla 1 se encuentran los datos descriptivos de la función sensorial y dependencia.

El $77.1 \%(f=74)$ presentó alteración del olfato y $77.1 \%(f=74)$ alteración del gusto. En la prueba de agudeza táctil, se encontró que $24.0 \%(f=23)$ de los adultos mayores presentó alteración en el pie izquierdo y el $30.2 \% \quad(f=29)$ en el pie derecho. En cuanto a la agudeza auditiva, $47.9 \%$ $(f=46)$ de los adultos mayores presentó audición normal en el oído izquierdo y el $42.7 \%$ $(f=41)$ en el oído derecho, en la tabla 2 se presenta la clasificación de acuerdo con el tipo de pérdida.

Respecto a la agudeza visual el $91.7 \%(f=88)$ resultó con alteración en la prueba sin anteojos. Del total de la muestra el 53.1\% $(f=51)$ refirió utilizar anteojos, por lo que se les hizo la prueba con anteojos y se encontró que de ellos el $70.6 \%$ $(f=36)$ presentó alteración. El 39.6\% $(f=38)$ resultaron con dependencia en las ABVD y el 24.0\% $(f=23)$ con dependencia en AIVD.

Con la prueba de correlación de Spearman se identificó relación estadísticamente significativa entre la agudeza del tacto en el pie izquierdo $\left(r_{s}\right.$ $=.266 ; p=.027)$ y derecho $\left(r_{s=}=230 ; p=.024\right)$ y la agudeza auditiva del oído derecho $\left(r_{\mathrm{s}}=-.258\right.$; $p=.011)$ con las ABVD. Así mismo, se observó que la edad $\left(r_{\mathrm{s}}=-.345 ; p=.001\right)$, agudeza del tacto en pie izquierdo $\left(\mathrm{r}_{\mathrm{s}}=.220 ; p=.031\right)$, auditiva del oído izquierdo $\left(r_{s}=-.283 ; p=.005\right)$ y derecho $\left(r_{s}=-.286 ; p=.005\right)$, olfato $\left(r_{s}=.241 ; p=.018\right)$ y gusto $\left(r_{s}=.203 ; p=.047\right)$ se relacionan con las AIVD (Tabla 3).

Para identificar si la dependencia para realizar las ABVD y AIVD es diferente en los adultos mayores con alteración visual en comparación con aquellos que tienen agudeza visual normal, se realizó la prueba $U$ de Mann-Whitney, con la que se observó que la dependencia para realizar las ABVD no fue diferente en la prueba sin anteojos $(U=127.500 ; \quad p=.079)$ como en la prueba con anteojos $(U=146.000 ; p=.754)$. Así mismo, se encontró que la dependencia para realizar las AIVD no fue diferente en la prueba sin anteojos $(U=160.000 ; p=.202)$ como en la prueba con anteojos $(U=115.000 ; p=.079)$.

Tabla 1. Datos descriptivos de edad, escolaridad, medicamentos utilizados, función sensorial y dependencia en actividades básicas e instrumentales.

\begin{tabular}{lcccc}
\hline Variable & Min & Max & Media & DE \\
\hline Edad & 60 & 84 & 69.33 & 6.53 \\
Escolaridad & 0 & 23 & 9.02 & 4.61 \\
Medicamentos & 0 & 10 & 3.31 & 2.07 \\
Monofilamento pie izquierdo & 0 & 10 & 9.27 & 1.77 \\
Monofilamento pie derecho & 1 & 10 & 9.17 & 1.83 \\
Audiometría oído izquierdo & 20 & 95 & 31.67 & 12.64 \\
\hline
\end{tabular}




\begin{tabular}{lcccc} 
Audiometría oído derecho & \multicolumn{2}{c}{} & 32.76 & 13.62 \\
Olfato & 15 & 50 & 2.98 & 1.56 \\
Gusto & 0 & 4 & 2.50 & 1.10 \\
ABVD & 0 & 100 & 95.68 & 8.00 \\
AIVD & 60 & 8 & 7.19 & 1.84 \\
\hline
\end{tabular}

Fuente: Resultados de Cédula de datos, de prueba de monofilamento, pruebas de gusto básico, prueba de aromas y agudeza auditiva, índice de Actividades básicas de la vida diaria e Instrumentales.

Tabla 2. Clasificación de la agudeza auditiva oído izquierda y derecho

\begin{tabular}{lccccc}
\hline & \multicolumn{2}{c}{ Oído izquierdo } & \multicolumn{2}{c}{ Oído derecho } \\
\multicolumn{1}{c}{ Categorías } & $\boldsymbol{f}$ & $\boldsymbol{\%}$ & $\boldsymbol{f}$ & \% \\
\hline Normal & 46 & 47.9 & 41 & 42.7 \\
Pérdida auditiva leve & 35 & 36.5 & 39 & 40.6 \\
Pérdida auditiva moderada & 9 & 9.4 & 11 & 11.5 \\
Pérdida auditiva moderadamente severa & 5 & 5.2 & 3 & 3.1 \\
Pérdida auditiva profunda & 1 & 1.0 & 2 & 2.1 \\
\hline Fuente: resultados de prueba auditiva. & & & & $n=96$
\end{tabular}

Tabla 3. Correlación de Spearman entre la función sensorial y actividades de la vida diaria

\begin{tabular}{lcccc}
\hline & \multicolumn{2}{c}{ ABVD } & \multicolumn{2}{c}{ AIVD } \\
\cline { 2 - 5 } & $\mathbf{r}_{\mathbf{s}}$ & $\boldsymbol{p}$ & $\mathbf{r}_{\mathbf{s}}$ & $\boldsymbol{p}$ \\
\hline Edad & -.066 & .522 & -.345 & $\mathbf{. 0 0 1}$ \\
Medicamentos & -.151 & .143 & -.097 & .347 \\
Monofilamento pie izquierdo & .226 & $\mathbf{. 0 2 7}$ & $\mathbf{. 2 2 0}$ & $\mathbf{. 0 3 1}$ \\
Monofilamento pie derecho &. $\mathbf{2 3 0}$ & $\mathbf{. 0 2 4}$ & .148 & .150 \\
Audiometría oído izquierdo & -.060 & .526 &.$- \mathbf{2 8 3}$ & $\mathbf{. 0 0 5}$ \\
Audiometría oído derecho & -.258 & $\mathbf{. 0 1 1}$ & $\mathbf{- . 2 8 6}$ & $\mathbf{. 0 0 5}$ \\
Olfato & .115 & .266 & $\mathbf{. 2 4 1}$ & $\mathbf{. 0 1 8}$ \\
Gusto & .065 & .529 & $\mathbf{. 2 0 3}$ & $\mathbf{. 0 4 7}$ \\
\hline Fuente: resuryyyyyy
\end{tabular}

Fuente: resultados de prueba de monofilamento, pruebas de gusto básico, prueba de aromas y agudeza auditiva, índice de Actividades básicas de la vida diaria e Instrumentales.

\section{DISCUSIÓN}

Se encontró que, en los sentidos del gusto y el olfato, más de tres cuartas partes de los adultos mayores que participaron en el estudio, resultaron con alteración, lo que indica que además de los cambios que ocurren en el envejecimiento y las enfermedades crónicas que los adultos padecen se alteran estos sentidos. No obstante, existen otros factores como la exposición a contaminantes, consumo de medicamentos y otras enfermedades que afectan el sentido del gusto y olfato, (3) dichos factores no fueron motivo de este estudio, por lo que se recomienda incluirlos en futuras investigaciones.

En relación con el tacto, aproximadamente una cuarta parte presentó afectación en la agudeza táctil en el pie izquierdo y más de una cuarta parte en el pie derecho. Este dato, coincide con lo reportado en la Encuesta ENSANUT, (2) donde se estudió a personas con diabetes mellitus, quienes reportaron que una de las 
complicaciones más frecuentes que han experimentado con la enfermedad es la pérdida de la sensibilidad en la planta de los pies, situación que resulta preocupante, debido a que la literatura indica que con el envejecimiento disminuye la función de los corpúsculos de meissner ubicados en las yemas de los dedos y piel del pie, lo que conlleva a la alteración de la percepción del tacto, (12) lo que pone en mayor riesgo a los adultos mayores que padecen enfermedades crónicas como la diabetes mellitus.

Respecto a la agudeza auditiva, más de la mitad de los adultos mayores presentaron alteración, pese a que existen pocos resultados con reportes de audición en adultos mayores con enfermedades crónicas, los hallazgos de este trabajo coinciden con lo encontrado en adultos sin enfermedad crónica. ${ }^{(13)}$ Lo que significa que las alteraciones en el sentido del oído se presentan por cambios del envejecimiento, donde las enfermedades crónicas como la diabetes mellitus, hipertensión y dislipidemias no tienen gran influencia; no obstante es necesario realizar más estudios que comprueben lo planteado y se explore el tipo de enfermedad crónica y tiempo de padecerla. En la valoración de la agudeza visual, casi todos los adultos mayores resultaron con alteración al realizar la prueba sin anteojos y un poco más de la mitad en la prueba con anteojos, este hallazgo es superior a lo reportado por otros autores. (13), (14), (15) y (16) Cabe mencionar que dichos estudios se realizaron en población abierta de adultos mayores, a diferencia del presente trabajo en el que se incluyeron exclusivamente adultos con enfermedades crónicas.

Los resultados encontrados respecto a la agudeza visual, se asemejan a lo reportado en la encuesta ENSANUT (2) respecto a las complicaciones que presentan las personas con diabetes mellitus, quienes mencionaron que la principal complicación fue la visión disminuida. Este hallazgo justifica la importancia de enfatizar en el autocuidado en adultos mayores con enfermedades crónicas, quienes a mayor edad presentan mayor probabilidad de pérdida visual por los cambios en el área interna de los ojos, como disminución de la musculatura de los párpados, iris, atrofia de las glándulas lagrimales y presión intraocular. (3)

Es importante resaltar que en la revisión de la literatura se encontró evidencia científica sobre la función sensorial en adultos mayores con diabetes mellitus principalmente, lo que limita la comparación de lo encontrado en el presente estudio donde se incluyeron adultos mayores con diferentes enfermedades crónicas. Así mismo, son pocos los estudios que se localizaron en los que se relaciona la función de la agudeza de los sentidos con las ABVD y las AIVD, lo que dificulta la comparación de lo encontrado en el presente trabajo. La literatura indica que algunas enfermedades crónicas alteran la agudeza olfativa y gustativa, (3) a su vez, dichas afecciones afectan la manera en que se realizan las actividades de la vida diaria, (17) sin embargo, los resultados de este estudio no mostraron relación estadísticamente significativa entre la agudeza olfativa y gustativa con las $A B V D$, únicamente se encontró que a mayor función olfativa y gustativa mayor independencia para realizar las AIVD; se piensa que la falta de significancia estadística pudiera estar relacionada con el tiempo de diagnóstico de la enfermedad, por lo que se sugiere que en futuros estudios se busque conocer la relación entre el tiempo de padecer la enfermedad y la alteración de la función sensorial.

En este sentido, los resultados de otro estudio (18) sugieren que la enfermedad crónica especialmente en las mujeres es mejor que en los hombres, ya que socialmente se espera que en el caso de las mujeres se encarguen de las tareas domésticas implicadas en las AIVD. Es así como, el enfoque de género se convierte en una limitante al contrastar la relación entre la función sensorial y la dependencia, debido a que los instrumentos empleados incluyen actividades de la vida diaria que en nuestro contexto social y culturalmente están asignadas a las mujeres como la preparación de la comida, cuidado de la casa y lavado de la ropa.

Se encontró que a mayor función del tacto mayor independencia para realizar las ABVD y las AIVD, este hallazgo se explica en que a través de este sentido es posible percibir las cualidades de los objetos; por lo que, cuando el adulto mayor no puede percibir las 
características de los objetos, presenta dificultad para realizar sus actividades cotidianas como bañarse o ducharse, preparar sus alimentos o el cuidado de la casa.

Además, se identificó que a menor audición mayor dependencia para realizar las ABVD y AIVD, este dato es congruente con hallazgos ${ }^{(19)}$ (20) quienes reportaron que la disfunción auditiva se asoció a mayor probabilidad de dependencia para realizar las actividades de la vida diaria, esta relación se debe a que los cambios en los órganos encargados de la audición pueden ocasionar deterioro del control postural ${ }^{(3)}$ y por lo tanto la movilización, lo que ocasiona dificultad para realizar las actividades cotidianas, ya que como afirma Aguilar y cols. (21) la movilización es elemental para realizar las actividades de vida.

Por último, se observó que la dependencia para realizar las ABVD y AIVD no es diferente en adultos mayores con alteración de la agudeza visual en comparación con aquellos que presentaron agudeza visual normal, este hallazgo es diferente de lo reportado por otros autores. (8) quienes encontraron evidencia estadísticamente significativa entre la agudeza visual y las actividades de la vida diaria.

\section{CONCLUSIONES}

En este estudio se comprobó empíricamente que a mayor función en los sentidos de tacto y oído, menor dependencia para realizar las ABVD y a mayor función del tacto, oído, olfato y gusto menor dependencia en las AIVD. Se observó que la dependencia en ABVD y AIVD no es diferente en los que presentaron alteración en la agudeza visual en comparación con los que resultaron normal. No obstante, es importante tener en cuenta que en este estudio se empleó un muestreo no probabilístico y un bajo tamaño de muestra, por lo que se recomienda tomar con cautela estos resultados.

Se considera necesario fortalecer y fomentar el autocuidado a través de intervenciones y educación del envejecimiento activo y saludable. La planeación de intervenciones a personas adultas mayores con enfermedades crónicas necesita considerar la función sensorial como factores que afectan la funcionalidad de este grupo poblacional, sobre todo porque la alteración de la función sensorial puede ser causa y consecuencia de enfermedades crónicas.

\section{Limitaciones}

Se desconoce la percepción de salud respecto a los sentidos por parte de los participantes y cuál sería la diferencia real en los participantes. No se verificó si existía alguna complicación propia de la enfermedad crónica que pudiera interferir con los resultados presentados.

\section{CONFLICTO DE INTERESES}

Los autores declararan no tener ningún tipo de

conflicto e intereses en relación al artículo.

\section{FINANCIAMIENTO}

Los autores declaran no haber recibido financiamiento para realizar este trabajo.

\section{REFERENCIAS BIBLIOGRÁFICAS}

1. Serra MA, Serra M, Viera M. Las enfermedades crónicas no transmisibles: magnitud actual y tendencias futuras.Revista Finlay, 2018; 8(2): 140-148.

2. Encuesta Nacional de Salud y Nutrición (ENSANUT). Encuesta Nacional de Salud y Nutrición de Medio Camino 2018. Informe final de resultados. México; [Internet]. INSP, SS. Disponible en https://www.gob.mx/cms/uploads/attachment/file/209093/ENSANUT. 
3. Eliopoulos C. Enfermería gerontológica. 8a ed. China: Wolters Kluwer; 2014. Disponible en: https://www.amazon.com.mx/Enfermer\%C3\%ADa-gerontol\%C3\%B3gica-Charlotte-Eliopoulosebook/dp/B01BKWN94U.

4. Carrillo B, Carrillo V, Astorga A, Hormachea D. Diagnóstico en la patología del olfato: Revisión de la literatura. Rev. Otorrinolaringol. Cir. Cabeza Cuello, [Internet].2017 [consultado Enero 2020]; 77(3):351360. Disponible en: https://scielo.conicyt.cl/pdf/orl/v77n3/0718-4816-orl-77-03-0351.pdf

5. Croy I, Nordin S, Hummel T. Trastornos olfativos y calidad de vida: una revisión actualizada. ChemSenses, [internet].2014; [consultado Enero 2020]; 39(3): 185-194.Disponible en: DOI Https://doi.org/10.1093/chemse/bjt072

6. López NC. El gusto por el sabor salado: perspectivas en nutrición humana. Perpect Nutr Humana. [Internet].2014; [consultado Enero 2020]16 (1): 99-109. Disponible en:

http://aprendeenlinea.udea.edu.co/revistas/index.php/nutricion/article/view/20315/17166

7. Del Castillo RA, Fernández JA, Del Castillo FJ. Guía de práctica clínica en el pie diabético. Med Pub Journals, [internet].2014; [consultado en Enero 2020]10 (2):1-17. Disponible en:

http://www.archivosdemedicina.com/medicina-de-familia/gua-de-prctica-clnica-en-el-pie-

diabtico.php?aid $=489$

8. Manrique-Espinoza B, Salinas-Rodríguez A, Moreno-Tamayo K M, Acosta-Castillo I, Sosa-Ortiz AL, Gutiérrez-Robledo LM. Téllez-Rojo MM. Condiciones de salud y estado funcional de los adultos mayores en México. Salud Pública Méx. 2013; 55: 323-331.

9. Rodríguez RM, Gaytán RP. Manual de audioprotesismo. [Internet]. 2006; [ consultado en enero 2020]; Disponible en: http://docplayer.es/6734210-Manual-de-audioprotesismo-dr-ruben-rodriguezmedrano-patricia-a-gaytan-rodriguez.html.

10. Cid-Ruzafa J, Damián-Moreno J. Valoración de la discapacidad física: el índice de Barthel. Rev. Esp. Salud Pública [Internet]. 1997 [citado 2018 Nov 26 ] ; 71( 2 ): 127-137. Disponible en: http://scielo.isciii.es/scielo.php?script=sci_arttext\&pid=\$1135-57271997000200004\&lng=es.

11. Trigás-Ferrín M, Ferreira-González L, Meijide-Míguez H. Escalas de valoración funcional en el anciano. [Internet] Galicia Clínica. 2011 [consultado diciembre 2010]; 72(1): 11-16. Disponible: https://meiga.info/escalas/valoracionfuncionaldelanciano.pdf

12. Porth CM. Fundamentos de fisiopatología. Mexico: Wolters Kluwer; 2011. [Internet] Disponible en: https://www.porrua.mx/libro/GEN:837004/fundamentos-de-fisiopatologia/carol-mporth/9788496921924

13. Cortés AR, Villarreal E, Galicia L, Martínez L, Vargas ER. Evaluación geriátrica integral del adulto mayor. Rev Med Chile. 2011; 139(6): 725-731. http://dx.doi.org/10.4067/S0034-98872011000600005

14. Cervantes RG, Villarreal E, Galicia L, Vargas E R, Martínez L. Estado de salud en el adulto mayor en atención primaria a partir de una valoración geriátrica integral. Aten Primaria. [Internet]. 2015 [consultado octubre 2019]; 47(6): 329-335. http://dx.doi.org/10.1016/j.

15. Gutiérrez JP, Rivera-Dommarco J, Shamah-Levy T, Villalpando-Hernández S, Franco A, Cuevas-Nasu L, Hernández-Ávila M. Encuesta Nacional de Salud y Nutrición: Resultados Nacionales. Cuernavaca, México: Instituto Nacional de Salud Pública 2012; Recuperado de 
http://ensanut.insp.mx/informes/ENSANUT2012ResultadosNacionales.pdf

16. Mora A, Mata F, Madrigal L. Guevara G. Frecuencia de baja visión en la población adulta mayor del distrito de San Isidro de El General, Costa Rica. Ciencia y Tecnología para la Salud Visual y Ocular, 2013; 11 (2): 27-41.

17. Hummel T, Nordin S. Olfactory disorders and their consequences for quality of life. Acta otolaryngologica [Internet] 2005 [consultado noviembre 2019]; 125 (2):116-121. Disponible en https://www.ncbi.nlm.nih.gov/pubmed/15880938.

18. Salazar-Barajas ME, Lillo CM, Hernández CPL, Villarreal RMA, Gallegos CE, Gómez MM, Salazar González BC. Factors Contributing to Active Aging in Older Adults, from the Framework of Roy's Adaptation Model. Invest. Educ. Enferm. 2018; 36(2):e08.

19. Semenov YR, Bigelow RT, Xue QL, Lac SD, Agrawal Y. Association between vestibular and cognitive function in US adults: data from the National Health and Nutrition Examination Survey. Journals of Gerontology Series A: Biomedical Sciences and Medical Sciences [Internet]2015 [consultado octubre 2019]; 71(2): 243-250. Disponible en:

https://academic.oup.com/biomedgerontology/article/71/2/243/2605620

20. Harun A, Semenov YR, Agrawal Y. Vestibular function and activities of daily living: analysis of the 1999 to 2004 National Health and Nutrition Examination Surveys. Gerontology and geriatric medicine [Internet] 2015 [consultado en marzo 2020]; disponible en:

https://www.ncbi.nlm.nih.gov/pubmed/26753170

21. Aguilar RM, Félix A, Vázquez L, Gutiérrez G, Martínez ML, Quevedo MM. El modelo de enfermería Roper-Logan-Tierney en el adulto mayor. México: McGrawHill; 2012. 